Article

\title{
Learning-Based Optimal Desired Compensation Adaptive Robust Control for a Flexure-Based Micro-Motion Manipulator
}

\author{
Shi Jia ${ }^{1,2}$, Yao Jiang ${ }^{3}$, Tiemin $\mathrm{Li}^{1,2, *}$ and Yunsong $\mathrm{Du}^{1,2}$ \\ 1 Institute of Manufacturing Engineering, Department of Mechanical Engineering, Tsinghua University, \\ Beijing 100084, China; thujiashi@163.com (S.J.); duyunsongwei@163.com (Y.D.) \\ 2 Beijing Key Lab of Precision/Ultra-precision Manufacturing Equipment and Control, Tsinghua University, \\ Beijing 100084, China \\ 3 Institute of Instrument Science and Technology, Department of Precision Instrument, Tsinghua University, \\ Beijing 100084, China; jiangyaonju@126.com \\ * Correspondence: litm@mail.tsinghua.edu.cn; Tel.: +86-10-6279-2792
}

Academic Editor: Chien-Hung Liu

Received: 12 March 2017; Accepted: 12 April 2017; Published: 17 April 2017

\begin{abstract}
Flexure-based micro-motion mechanisms activated by piezoelectric actuators have a wide range of applications in modern precision industry, due to their inherent merits. However, system performance is negatively affected by model uncertainty, disturbance and uncertain nonlinearity, such as the cross-coupling effect and the hysteresis of the actuator. This paper presents an integrated learning-based optimal desired compensation adaptive robust control (LODCARC) methodology for a flexure-based parallel micro-motion manipulator. The proposed LODCARC optimizes the reference trajectory used in the desired compensation adaptive robust control (DCARC) by iterative learning control (ILC), which can greatly compensate for the effect of repetitive disturbance and uncertainty. The proposed control approach was tested on the flexure-based micro-motion manipulator, with the comparative results of high-speed tracking experiments verifying that the proposed LODCARC controller can achieve excellent tracking and contouring performances with parametric adaption and disturbance robustness. Furthermore, the iterative reference optimization can effectively accommodate the effects of unmodeled repetitive uncertainty from the micro-motion system. This study provides a practical and effective technique for the flexure-based micro-motion manipulator to achieve high-precision motion.
\end{abstract}

Keywords: flexure-based manipulator; precision micro-motion; desired compensation adaptive robust control; reference optimization

\section{Introduction}

There are a wide variety of mechanisms such as compliant mechanism and parallel mechanism for high-precision positioning and motion technology [1-3]. Flexure-based compliant mechanisms, which transmit the motions entirely through the deformation of materials, exhibit the advantages of a typical micro-motion mechanism in terms of no backlash, no wear, and easy manufacturing [4]. Both serial and parallel structures have been adopted in flexure-based mechanisms, although most flexure-based stages are developed based on a parallel structure. This is due to the fact that parallel flexure-based stages have advantages of high rigidity and frequency, low inertia and symmetrical characteristics of each axis [5-7]. A piezoelectric actuator is usually adopted as the actuator of the flexure-based manipulator due to its merits of high-precision, fast response, high stiffness and large force [8]. However, some dynamical behaviors of micro-motion system are challenging from the 
perspective of control. In particular, there is inevitable model uncertainty and nonlinearity caused by cross-axis coupling effects, rigidity variation in addition to the hysteresis and other nonlinearity of the piezoelectric actuator $[9,10]$, which will obviously deteriorate motion accuracy, especially in high-speed applications.

Therefore, the controller design plays an important role in achieving great tracking and contouring performance of flexure-based micro-motion manipulator. For general purposes, a simple proportional-integral-derivative (PID) controller is always applied in the flexure-based micro-motion stage [11]. However, due to increasing demands for higher speeds from the micro-motion manipulator, the PID controller cannot efficiently deal with the model uncertainty and nonlinear effect of the system, resulting in its limited performance. Subsequently, model-based feedforward controllers have been developed. In a previous study [12], an inversion-based feedforward controller combined with a PID feedback controller was utilized to compensate for the nonlinearity of a parallel micro-positioning stage, with good positioning and tracking performances having been achieved. Furthermore, different types of mathematical models, such as the Prandtl-Ishlinskii model [13], Presiach model [14] and Bouc-Wen model [15], have been applied for feedforward control in order to compensate the hysteresis of piezoelectric actuator. It is noted that, when the micro-motion stage is used for micro machining, the cutting forces should be considered for achieving smooth motion and high performance machining [16,17]. However, the dynamics of the system are complicated so the accuracy of the mathematical model used in feedforward control is always limited. Furthermore, there inevitably exists model uncertainty and perturbation. For repetitive tracking tasks, iterative learning control (ILC) [18] can be adopted to compensate for the repetitive uncertainty and disturbance by using information from previous executions. In a previous study [19], ILC was used to compensate for the hysteresis of the piezoelectric actuator while too many iteration trials had to be taken in this study to get the accurate dynamical model of the system and achieve excellent tracking performance, which is too long for real-world applications. In any case, ILC is sensitive to parametric uncertainty and uncertain disturbance [18]. One typical method to deal with the model uncertainty and disturbance is adaptive robust control [20-22], which is widely used in many industrial applications. However, the velocity measurement noise greatly reduces the performance of adaptive robust control, especially in flexure-based micro-motion stages with high rigidity where velocity measurement noise is relatively large. To reduce the effect of measurement noise, a desired compensation adaptive robust control (DCARC) [23,24] was presented. The DCARC strategy is constructed based on a dynamical model of the system with prior knowledge of the bounds of the parametric uncertainties and the bounds of the unmodeled uncertainty and nonlinearity as well as disturbance. The parameters are adjusted on-line via certain parameter adaptation law to achieve an improved model compensation, while the model uncertainty and lumped disturbance are handled by certain robust law. Thus, the DCARC possesses the advantages of both control methods, which has a parametric adaptation ability and guaranteed performance to the model uncertainty and disturbance. In addition, compared with conventional adaptive robust control, the adaptive model compensation part of DCARC depends on the desired reference trajectory instead of actual state measurements, so the effect of measurement noise is minimized.

However, the effects of unmodeled dynamics of the plant are suppressed by the robust control term in DCARC, so the final tracking performance is unavoidably conservative. In this paper, an optimization control method of DCARC, learning-based optimal desired compensation adaptive robust control (LODCARC) is proposed and implemented for a flexure-based parallel micro-motion stage. The stage is activated by piezoelectric actuators in $X$ - and $Y$-axes. It has a decoupled symmetrical structure so the multi-input multi-output (MIMO) system can be simplified as two single-input single-output (SISO) sub-systems, with the proposed LODCARC being implemented in each sub-system. The DCARC term of the LODCARC is designed based on the dynamics of the micro-motion system under the consideration of parametric uncertainty and uncertain nonlinearity. Furthermore, the effect of measurement noise is minimized in DCARC by using the desired reference trajectory rather 
than actual state measurements. However, considering the complicated environment, the accurate dynamical model of flexure-based micro-motion system is difficult to obtain and for micro-scale motion, many motion tasks are repetitive in a finite time. Therefore, ILC was used to optimize the reference trajectory used in the DCARC term to compensate for the effects of unmodeled repetitive uncertainty and nonlinear disturbance. The whole control scheme with the stability analysis is presented and comparative experimental investigation of high-speed trajectory is carried out, with the experimental results showing that the tracking and contouring performances of the flexure-based micro-motion stage are greatly improved by adopting the proposed LODCARC.

\section{Description of the Investigated Flexure-Based Manipulator}

The parallel flexure-based micro-motion manipulator investigated in this paper is schematically illustrated in Figure 1a and the manufactured prototype of the manipulator, which is monolithically milled from a block of Al-7075, is shown in Figure 1b. As shown in Figure 1b, the piezoelectric actuators (PSt 150/7/40, VS 12, XMT Harbin, China) adopted for the system have a $40 \mu \mathrm{m}$ range, while the output displacements of the terminal platform along the $X$ - and $Y$-axes are measured by two high precision length gauges (MT-1281, Heidenhain, Bavaria, Germany). To realize high stiffness, high natural frequency and output decoupling characteristic of the micro-motion manipulator, this study used a symmetrical parallel structure composed of four identical limbs $[25,26]$. Each limb is composed of a parallelogram flexure and a fixed-fixed beam, which are serially connected and acted as prismatic joints. Therefore, the micro-motion stage has two translational degrees of freedom along the $X$ - and $Y$-axes.

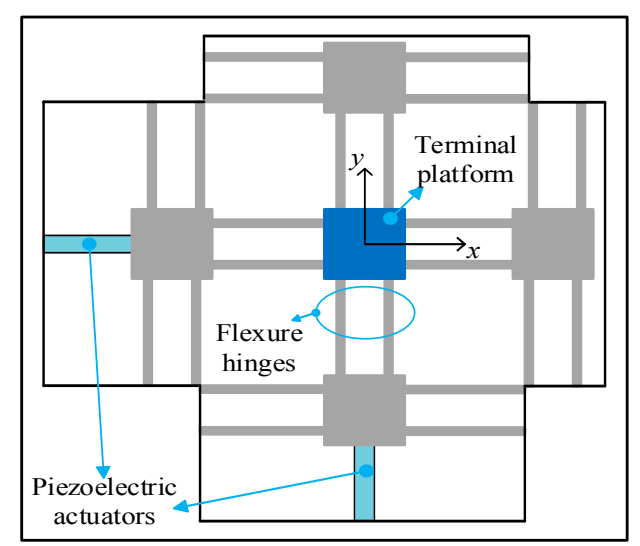

(a)

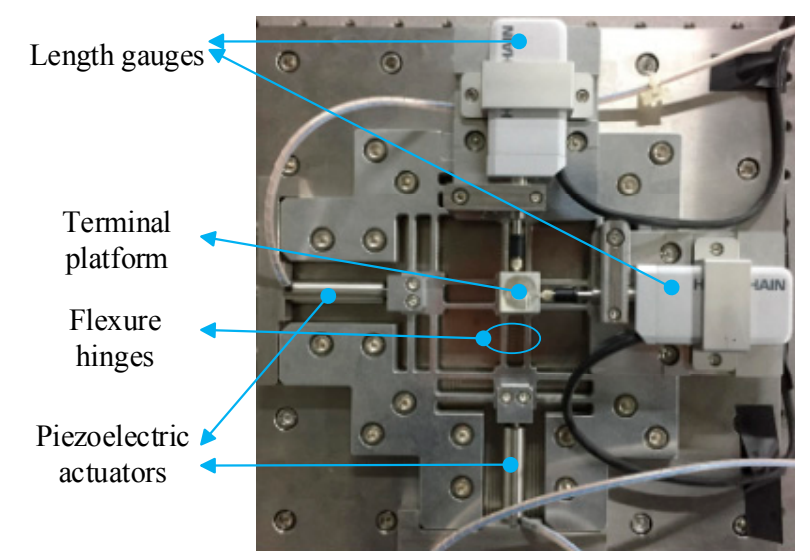

(b)

Figure 1. The parallel flexure-based micro-motion manipulator. (a) Schematic diagram; (b) Manufactured prototype.

Due to the symmetrical structure of the micro-motion stage, it was necessary to study only the dynamics of the stage along the $X$-axis. The dynamic model of the mechanical part of the system is commonly treated as a second-order system according to the characteristics of its mechanical structure [27] and can be expressed as follows:

$$
m_{e} \ddot{x}(t)+c_{e} \dot{x}(t)+k_{e} x(t)=F_{p}(t)+f_{d}(t)+\Delta
$$

where $m_{e}, c_{e}$, and $k_{e}$ are the moving mass, damping coefficient and stiffness of the stage, respectively; $x$ is the output displacement of the terminal platform along the $X$-axis; $F_{p}$ is the driving force of the piezoelectric actuator; $f_{d}$ is the external disturbance and $\Delta$ is the lumped uncertain of the system. As the power amplifier of the piezoelectric actuator has a very high bandwidth, it is reasonable to assume that the dynamics from the input voltage to the output displacement of the piezoelectric actuator 
can be simplified as a constant gain $\gamma$. Thus, we can note that $F_{p}=\gamma k_{i n} u$, where $k_{\text {in }}$ is the input axial stiffness of the micro-motion stage and $u$ is the control input voltage.

Subsequently, the dynamic model of the whole stage can be derived as a second-order system.

$$
\theta_{1} \ddot{x}+\theta_{2} \dot{x}+\theta_{3} x=u+\theta_{4}+d
$$

where $\theta_{1}=m_{e} /\left(\gamma k_{\text {in }}\right), \theta_{2}=c_{e} /\left(\gamma k_{\text {in }}\right), \theta_{3}=k_{e} /\left(\gamma k_{\text {in }}\right), \theta_{4}=f_{d} /\left(\gamma k_{\text {in }}\right)$ and $d=\Delta /\left(\gamma k_{\text {in }}\right)$. The unknown parameter set is defined as $\theta=\left[\theta_{1}, \theta_{2}, \theta_{3}, \theta_{4}\right]^{\mathrm{T}}$, with an open-loop sinusoidal sweeping test subsequently being carried out to estimate the parameters in Equation (2). The sweeping sinusoidal signals ranging from $1 \mathrm{~Hz}$ to $1500 \mathrm{~Hz}$, with the sampling frequency of $20 \mathrm{kHz}$, are used as the control input to excite the system. The system identification tool of Matlab is used to process data and estimate the model by using the response data from experiments, with the parameter estimates in $X$ - and $Y$-axes direction being $\theta_{x}=\left[6.35 \times 10^{-7}, 5.95 \times 10^{-3}, 6.23,0\right]^{\mathrm{T}}$ and $\theta_{y}=\left[5.20 \times 10^{-7}, 6.10 \times 10^{-3}, 6.65,0\right]^{\mathrm{T}}$, respectively. The following practical assumption can be made.

Assumption 1: The extent of the parametric uncertainties and uncertain nonlinearities are known, namely:

$$
\begin{gathered}
\theta \in \Omega_{\theta} \triangleq\left\{\theta: \theta_{\min } \leq \theta \leq \theta_{\min }\right\} \\
d \in \Omega_{d} \triangleq\left\{d:|d| \leq \delta_{d}\right\}
\end{gathered}
$$

where $\theta_{\min }=\left[\theta_{1 \min }, \theta_{2 \min }, \theta_{3 \min }, \theta_{4 \min }\right]^{T}, \theta_{\max }=\left[\theta_{1 \max }, \theta_{2 \max }, \theta_{3 \max }, \theta_{4 \max }\right]^{T}$, and $\delta_{d}$ are known.

\section{Control System Design for the Flexure-Based Manipulator}

Figure 2 depicts the whole control structure of LODCARC for flexure-based micro-motion stage to achieve ultra-high precision motion. The DCARC term is designed based on the system dynamics that aim to achieve parameter adaption and certain robustness, although in real-world practice with the complicated environment, there exists unavoidable tracking error, which is defined as $e=x-x_{d}$. Furthermore, the unmodeled uncertainty and disturbance affecting the tracking performance of the micro-motion stage are repetitive, to a large extent. The ILC optimization term is treated as a trajectory optimization to alter the reference for DCARC. The optimal signal $x_{\text {opt }}$ of ILC is based on previous repetitive control information including tracking error $e$, which aims to compensate for the effects of unmolded repetitive uncertainty and disturbance. Thus, with reference optimization by ILC (Figure 2), the reference trajectory used for DCARC is adjusted as $x_{a}=x_{d}+x_{o p t}$, with the accuracy of the flexure-based micro-motion stage able to be further improved.

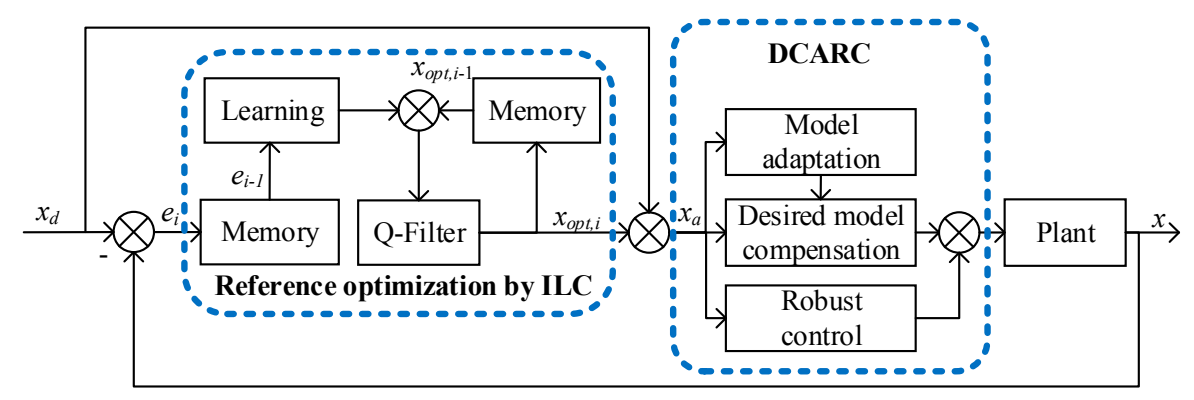

Figure 2. Structure of the control system for the flexure-based micro-motion manipulator.

\subsection{DCARC Design in LODCARC}

As mentioned, DCARC has a parametric adaptation ability of adaptive control and having robust performance to the uncertainty and disturbance of robust control. The design of the DCARC term of the proposed LODCARC for the flexure-based system is as follows: Define $\hat{\theta}$ as the estimate of $\theta$, then 
the estimation error of $\theta$ can be defined as $\widetilde{\theta}=\hat{\theta}-\theta$. In the DCARC term, the estimate $\hat{\theta}$ of parameter is updated by using the following discontinuous projection type adaptation law:

$$
\dot{\hat{\theta}}=\operatorname{Proj}_{\hat{\theta}}(\Gamma \tau)
$$

where $\Gamma$ is a diagonal positive definite matrix, which indicates the adaptation rate, and $\tau$ is an adaptation function. The projection mapping $\operatorname{Proj}_{\hat{\theta}}(\bullet)=\left[\operatorname{Proj}_{\hat{\theta}_{1}}\left(\bullet_{1}\right), \cdots, \operatorname{Proj}_{\hat{\theta}_{4}}\left(\bullet_{4}\right)\right]^{\mathrm{T}}$ is defined by

$$
\operatorname{Proj}_{\hat{\theta}_{i}}\left(\bullet_{i}\right)=\left\{\begin{array}{l}
0, \text { if } \hat{\theta}_{i}=\theta_{i \text { max }} \text { and } \bullet_{i}>0 \\
0, \text { if } \hat{\theta}_{i}=\theta_{\text {imin }} \text { and } \bullet_{i}<0 \\
\bullet_{i}, \text { otherwise }
\end{array}\right.
$$

It can be shown [28] that for any adaptation function $\tau$, the projection mapping defined in Equation (6) guarantees

$$
\begin{aligned}
& \text { P1 : } \hat{\theta} \in \Omega_{\theta} \triangleq\left\{\hat{\theta}: \theta_{\min } \leq \hat{\theta} \leq \theta_{\max }\right\} \\
& \text { P2: } \widetilde{\theta}^{\mathrm{T}}\left(\Gamma^{-1} \operatorname{Proj}_{\hat{\theta}}(\Gamma \tau)-\tau\right) \leq 0, \forall \tau
\end{aligned} .
$$

After this, define a switching-function-quantity as

$$
p=\dot{e}_{a}+k_{1} e_{a}=\dot{x}-x_{e q}, x_{e q} \triangleq \dot{x}_{a}-k_{1} e_{a}
$$

where $e_{a}=x-x_{a}=x-\left(x_{d}+x_{o p t}\right)$ is the adjusted tracking error; $x, x_{d}$ and $x_{a}$ are the actual output, desired reference and adjusted optimized reference, respectively; And $k_{1}$ is a positive feedback gain. Differentiating Equation (8) and noting Equation (2), one can obtain

$$
\begin{aligned}
\theta_{1} \dot{p} & =u-\theta_{1} \dot{x}_{e q}-\theta_{2} \dot{x}-\theta_{3} x+\theta_{4}+d \\
& =u+\varphi^{\mathrm{T}} \theta+d
\end{aligned}
$$

where $\varphi=\left[-\dot{x}_{e q},-\dot{x},-x, 1\right]^{\mathrm{T}}$. Define $\varphi_{a}=\left[-\ddot{x}_{a},-\dot{x}_{a},-x_{a}, 1\right]^{\mathrm{T}}$ and the DCARC control law is proposed as

$$
u=u_{f}+u_{s}
$$

where $u_{f}=-\varphi_{a}^{\mathrm{T}} \hat{\theta}$ is the adjustable model compensation term for perfect trajectory tracking. It should be noted here that the adjustable model compensation is decided by adjusted optimized reference and parameter estimations only, rather than the actual output, which has great velocity measurement noise. In this way, DCARC can minimize the effect of measurement noise compared with conventional adaptive robust control. Furthermore, $u_{s}$ is the feedback control term, which will be synthesized later. Substituting Equation (10) into Equation (9) and then simplifying the resulting expression leads to

$$
\theta_{1} \dot{p}=u_{s}-\varphi_{a}{ }^{\mathrm{T}} \widetilde{\theta}+d+\left(k_{1} \theta_{1}-\theta_{2}\right) \dot{e}_{a}-\theta_{3} e_{a}
$$

In addition, the feedback control term $u_{s}$ in Equation (10) consists of two terms given by

$$
u_{s}=u_{s 1}+u_{s 2}
$$

where $u_{s 1}=-k_{s 1} p$ is used to stabilize the nominal system and $u_{s 2}$ is a robust feedback used to attenuate the effect of model uncertainties. For traditional adaptive robust control, $u_{s 1}$ is just a simple proportional feedback with $k_{s}$ being the feedback gain to stabilize the nominal system [24], while in this case for DCARC, $k_{s 1}$ in Equation (12) is required to be large enough such that the matrix $A$ defined below is positive definite.

$$
A=\left[\begin{array}{cc}
k_{s 1}-k_{s}-k_{1} \theta_{1}+\theta_{2} & -\frac{k_{1} \theta_{2}-\theta_{3}}{2} \\
-\frac{k_{1} \theta_{2}-\theta_{3}}{2} & \frac{m_{e} k_{1}^{3}}{2}
\end{array}\right] .
$$


Furthermore, $u_{s 2}$ as a robust feedback term is designed to attenuate the effect of model uncertainties of parametric uncertainty and uncertain nonlinearity. As such, $u_{s 2}$ should dissipate naturally so that it does not affect the adaptive function of the adaptive control part $u_{f}$. Thus, noting Assumption 1 and part 1 of Equation (7), there is a $u_{s 2}$ to satisfy the following constraints:

$$
\begin{array}{ll}
\text { i } & p\left\{u_{s 2}-\varphi_{a} \mathrm{~T} \widetilde{\theta}+d\right\} \leq \varepsilon \\
\text { ii } & p u_{s 2} \leq 0
\end{array}
$$

where $\epsilon$ is a design parameter, which can be arbitrarily small. Specific form of $u_{s 2}$ can be obtained using the techniques in [20]:

$$
u_{s 2}=-\frac{1}{4 \varepsilon} h^{2} p
$$

where $h$ is any function satisfying

$$
h \geq\left\|\theta_{M}\right\|\left\|\varphi_{d}\right\|+\delta_{d}
$$

where $\theta_{\mathrm{M}}=\theta_{\max }-\theta_{\min }$.

Theorem 1: If the adaptation function in Equation (5) is chosen as

$$
\tau=\varphi_{a} p
$$

then the DCARC control term applied in Equation (5) guarantees the following results.

1. In general, all signals are bounded, and the positive definite function $V_{s}$ defined by

$$
V_{s}=\frac{1}{2} \theta_{1} p^{2}+\frac{1}{2} \theta_{1} k_{1}^{2} e_{a}^{2}
$$

is bounded above by

$$
V_{s} \leq \exp (-\lambda t) V_{s}(0)+\frac{\varepsilon}{\lambda}[1-\exp (-\lambda t)]
$$

where $\lambda=\min \left\{2 k_{s} / \theta_{1 \max }, k_{1}\right\}$.

2. If after a finite time $t_{0}$, there exists parametric uncertainties only (i.e., $d=0$ and $\forall t \geq t_{0}$ ), then zero final tracking error is also achieved, i.e., $e_{a} \rightarrow 0$ and $p \rightarrow 0$ as $t \rightarrow \infty$.

Proof. Differentiating $V_{s}$ given by Equation (18) with respect to time and applying Equation (11) yields

$$
\dot{V}_{s}=p\left\{u_{s}-\varphi_{a}{ }^{\mathrm{T}} \widetilde{\theta}+d+\left(k_{1} \theta_{1}-\theta_{2}\right) \dot{e}_{a}-\theta_{3} e_{a}\right\}+\theta_{1} k_{1}^{2} e_{a} \dot{e}_{a}
$$

Noting $p=\dot{e}_{a}+k_{1} e_{a}$, we have

$$
\begin{aligned}
\dot{V}_{s} & =p\left\{u_{s 2}-\varphi_{a}{ }^{\mathrm{T}} \widetilde{\theta}+d\right\}+\left(k_{1} \theta_{1}-\theta_{2}-k_{s 1}\right) p^{2}+\left(k_{1} \theta_{2}-\theta_{3}\right) e_{a} p-\theta_{1} k_{1}{ }^{3} e_{a}{ }^{2} \\
& =p\left\{u_{s 2}-\varphi_{a}{ }^{\mathrm{T}} \widetilde{\theta}+d\right\}-\left[p, e_{a}\right] A\left[p, e_{a}\right]^{\mathrm{T}}-k_{s} p^{2}-\frac{1}{2} \theta_{1} k_{1}{ }^{3} e_{a}{ }^{2}
\end{aligned}
$$

As $A$ is positive definite, then we have

$$
\dot{V}_{s} \leq p\left\{u_{s 2}-\varphi_{a}{ }^{\mathrm{T}} \widetilde{\theta}+d\right\}-k_{s} p^{2}-\frac{1}{2} \theta_{1} k_{1}{ }^{3} e_{a}{ }^{2}
$$

With condition i of Equation (14) and choosing $\lambda$ as $\lambda=\min \left\{2 k_{s} / \theta_{1 \max }, k_{1}\right\}$, the derivative of $V_{s}$ becomes

$$
\dot{V}_{s} \leq-\lambda V_{s}+\varepsilon
$$

Thus, part 1 of Theorem 1 is proved. 
Considering the situation in part 2 of Theorem 1, i.e., $d=0$ and $\forall t \geq t_{0}$, choose a positive definite function $V_{\theta}$ as

$$
V_{\theta}=V_{s}+\frac{1}{2} \widetilde{\theta}^{\mathrm{T}} \Gamma^{-1} \widetilde{\theta}
$$

From Equation (22), the derivative of $V_{\theta}$ satisfies

$$
\dot{V}_{\theta} \leq p\left\{u_{s 2}-\varphi_{a}^{\mathrm{T}} \widetilde{\theta}+d\right\}-k_{s} p^{2}-\frac{1}{2} \theta_{1} k_{1}{ }^{3} e_{a}{ }^{2}+\widetilde{\theta}^{\mathrm{T}} \Gamma^{-1} \dot{\hat{\theta}}
$$

As $d=0$ and $\forall t \geq t_{0}$, we have

$$
\dot{V}_{\theta} \leq p u_{s 2}+\widetilde{\theta}^{\mathrm{T}}\left(\Gamma^{-1} \operatorname{Proj}_{\hat{\theta}}(\Gamma \tau)-\tau\right)-k_{s} p^{2}-\frac{1}{2} \theta_{1} k_{1}^{3} e_{a}^{2}
$$

From condition ii of Equation (14) and part 2 of Equation (8), $\dot{V}_{\theta}$ satisfies

$$
\dot{V}_{\theta} \leq-k_{s} p^{2}-\frac{1}{2} \theta_{1} k_{1}^{3} e_{a}^{2}
$$

Let $-k_{s} p^{2}-\frac{1}{2} \theta_{1} k_{1}{ }^{3} e_{a}^{2}=-M$. Therefore, $V_{\theta} \in L_{\infty}$ and $M \in L_{1}$. As all signals are bounded, it is easy to check that $\dot{M}$ is bounded, thus making $M$ uniformly continuous. By Barbalat's lemma, $M \rightarrow 0$ as $t \rightarrow \infty$, resulting in zero final tracking error being achieved.

\subsection{Reference Optimization in LODCARC}

The proposed DCARC term in Section 3.1 is designed to track $x_{a}=x_{o p t}+x_{d}$ as accurately as possible, which also means if $x_{\text {opt }}=0$, DCARC term can track the desired reference $x_{d}$ as accurately as possible. Furthermore, Theorem 1 reveals the stability of the result, even resulting in a zero tracking error. However, in real-world applications, tracking error is inevitable (i.e., $e_{a}=x-x_{a}$ cannot be zero) and in practice, there exists residual tracking error of the practical motion control system $e_{a}$. As $e_{a}=x-\left(x_{\text {opt }}+x_{d}\right)=e-x_{\text {opt }}$, we have

$$
e=e_{a}+x_{o p t}
$$

Thus, if the optimal input signal $x_{\text {opt }}$ could capture the characteristics of $-e_{a}$ (i.e., $x_{\text {opt }} \rightarrow e_{a}$ ), the actual tracking error $e$ could achieve $e \rightarrow 0$. Therefore, iterative learning control (ILC), which can improve tracking performance by using information from previous executions to compensate for the repetitive uncertainty and perturbation, is used to capture the characteristics of the residual tracking error. As shown in Figure 2, the ILC term is used to generate the optimal input signal for adjusting the desired reference of DCARC. Denote the dynamics of the closed-loop of DCARC with the flexure-based plant as $P(\mathrm{~s})$, then the whole control system can be derived as

$$
X_{i}(s)=P(s)\left[X_{o p t, i}(s)+X_{d}(s)\right]
$$

where subscript $i$ denotes the iteration trial number, while the optimal input signal $X_{o p t, i}$ is generated by a iterative learning law as

$$
X_{o p t, i}(s)=Q(s)\left(X_{o p t, i-1}(s)+L(s) E_{i-1}(s)\right)
$$

where $E_{i-1}$ is the tracking error in the $(i-1)$ th iterative process, $Q$ is a low-pass $Q$-filter which is utilized to enhance the system robustness and suppress the noise in the iterative process, while $L$ is the learning function. It is noted that a low-pass filter may cause a phase shift, although the phase shift can be eliminated by filtering the signal back and forth [29]. Many types of learning functions have been 
developed for ILC [18], while PD-type learning function is a typical, simple and tunable ILC learning function. PD-type learning function was chosen for this paper and it is written as

$$
L(s)=K_{p}+K_{d} s
$$

Following this, asymptotic stability and monotonic convergence in the iteration domain of the iterative law can be guaranteed under certain conservative conditions, which are presented in [18] as the following Lemma.

Lemma 1: The iterative learning law defined in Equations (30) and (31) acting on the system $P(s)$ is monotonically convergent i.e., $\left\|E_{\infty}(s)-E_{i+1}(s)\right\|_{\infty}<\alpha\left\|E_{\infty}(s)-E_{i}(s)\right\|_{\infty}$, where $\alpha$ is the convergence rate, if

$$
\|Q(s)(1-L(s) P(s))\|_{\infty}<\alpha<1
$$

Based on Lemma 1, the optimal input signal $x_{\text {opt }}$ generated by ILC control can capture the characteristics of residual tracking errors caused by unmodeled repetitive uncertainty. If the convergence of ILC is guaranteed, the optimal input signal $x_{\text {opt }}$ will be determined and then according to Theorem 1 , the stability of the whole control system can be guaranteed. Furthermore, as mentioned, zero tracking error is achieved if there are only parametric uncertainties so $P(s)$ can be considered as $P(s)=1$ theoretically. After this, the convergence conditions can be obtained by substituting Equation (31) into Equation (32) to obtain

$$
\left\|Q(s)\left(1-K_{p}+K_{d} s\right)\right\|_{\infty}<\alpha<1
$$

Obviously, if $K_{p}+K_{d} s=1$ (i.e., $K_{p}=1$ and $K_{d}=0$ ), then we could obtain $\alpha=0$ in theory, which means the ILC term will reach a converged error value after one iteration.

\section{Experimental Verification}

\subsection{Experiment Setup}

To validate the proposed control method, the comparative experiments were carried out on the flexure-based micro-motion manipulator as depicted in Figure 1, while the whole experimental system is established as depicted in Figure 3. The two actuators are controlled by a modular piezo-servo controller (XE500/E50, XMT Harbin, China). A dSPACE processor board DS1007 equipped with a 16-bit ADC card (DS2102) and a 6-channel high resolution incremental encoder interface card (DS3002) is utilized to output the excitation voltage of the piezo servo controller and capture the real-time data for the length gauges. Since the piezoelectric actuator is made up of multiple piezoelectric layers glued together, it is sensitive to the pulling force, which may bring damage to the actuator. Therefore, preloading forces were applied on the two piezoelectric actuators by tightening the bolts to guarantee their operational safety.

To show the effectiveness of the proposed LODCARC sufficiently, three more controllers, namely the PI, ILC and DCARC controllers, were implemented for comparison. A traditional PI controller, as a typical kind of PID controller, consists of a proportional term and an integral term, which is simple and widely-used. On the other hand, an individual ILC controller uses the same learning function in the proposed learning-based optimal DCARC controller as discussed above in Section 3.2. It was noted that a second order low-pass with the expression of $Q(s)=\frac{\omega_{n}^{2}}{s^{2}+2 \zeta \omega_{n} s+\omega_{n}^{2}}$ is used for the $Q$-filter in an ILC controller, where $\zeta$ is damping ratio and $\omega_{n}$ is the crossover frequency. In this case, $\zeta=0.7$ and $\omega_{n}=5026.55 \mathrm{rad} / \mathrm{s}$. The PD-type learning function of ILC controller, as mentioned in Equation (31), was chosen as $K_{p}=4.5$ and $K_{d}=3.0$. As for the DCARC controller, the boundaries for the variation in parameters in the $X$-axis were chosen as $\theta_{x \min }=\left[6.13 \times 10^{-7}, 5.90 \times 10^{-3}, 6.10,-2\right]^{\mathrm{T}}$ and $\theta_{x \max }=\left[6.38 \times 10^{-7}, 6.10 \times 10^{-3}, 6.55,2\right]^{\mathrm{T}}$, while the boundaries for the variation in parameters in the Y-axis are chosen as 
$\theta_{y \min }=\left[5.15 \times 10^{-7}, 5.90 \times 10^{-3}, 6.50,-6\right]^{\mathrm{T}}$ and $\theta_{y \max }=\left[5.29 \times 10^{-7}, 6.20 \times 10^{-3}, 6.80,6\right]^{\mathrm{T}}$. The diagonal positive definite matrix $\Gamma$, which indicates the adaptation rate in Equation (5) is chosen as $\Gamma=\operatorname{diag}\left[1.50 \times 10^{-16}, 5.00 \times 10^{-9}, 5.00 \times 10^{-3}, 1.75 \times 10^{-1}\right]$. It was noted that the DCARC term of the proposed learning-based optimal DCARC controller and the DCARC controller introduced above were the same. In addition, to make sure that the comparative experiments were accurate and fair, the proposed LODCARC controller used the same control parameters as a DCARC controller and the same filter parameters as an individual ILC controller.

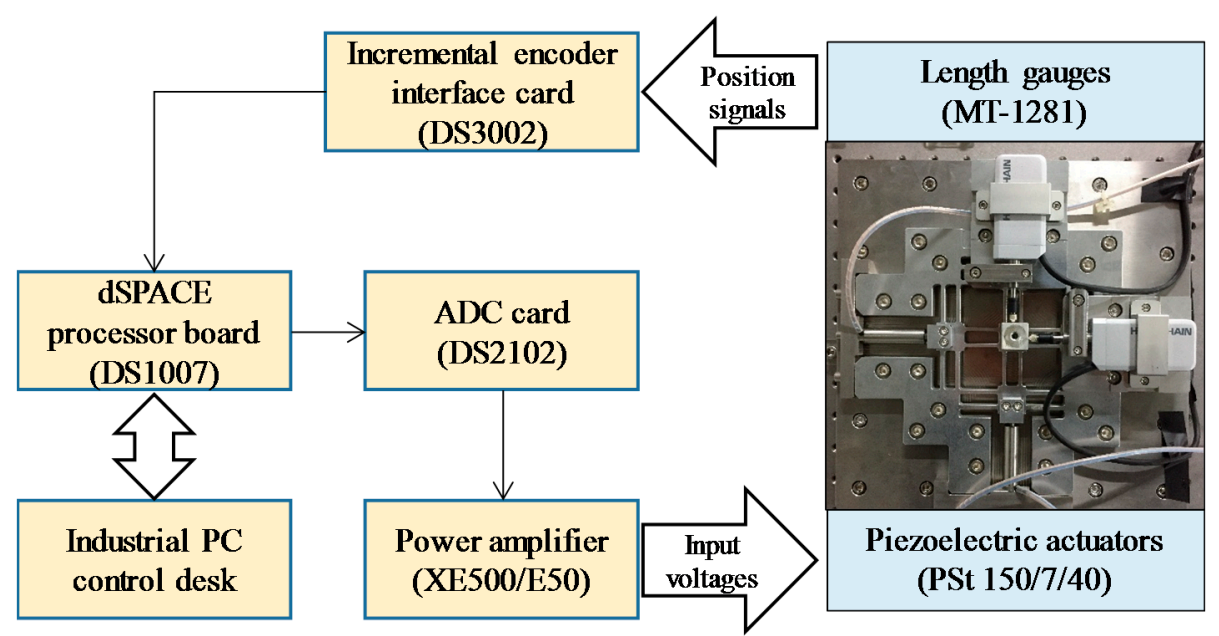

Figure 3. Experiment setup.

\subsection{Experimental Results}

\subsubsection{High-Speed Circular Contouring Trajectory}

A high-speed circular contouring trajectory with a radius of $5 \mu \mathrm{m}$ and a frequency of $200 \mathrm{~Hz}$ was chosen to test the effectiveness of the proposed control method first. To get a circular trajectory, sinusoidal signals were adopted as input signals in the $X$ - and $Y$-axes with the phase quadrature. As each axis tracks a sinusoidal trajectory when the micro-motion stage tracks a circular trajectory, the tracking performance in one direction was considered first. Figure 4 shows the tracking errors in the $X$-axis over $0.2 \mathrm{~s}$ using four control methods. In addition, two performance indexes, namely the root mean square value of the tracking error $\left(e_{R M S}\right)$ and the maximum value of the tracking error $\left(e_{R M S}\right)$, were introduced to evaluate the performances of different controllers mentioned above, with the performance indexes of the tracking error in Figure 4 being compared in Table 1.

It can be observed from Figure 4 that the tracking performance of PI controller in the $X$-axis is the worst, due to the phase lag problem of PI controller under high-speed motion conditions. In comparison, the other controllers can achieve good tracking performances. However, the dynamics of the stage in $X$ - and $Y$-axes are identical theoretically due to the symmetrical structure of the micro-motion stage. As a result, the phase lag in two directions is the same for the circular trajectory, which will lead to a relatively good contouring performance. As for the other three controllers, the $e_{R M S}$ of the ILC and DCARC controllers were above $0.13 \mu \mathrm{m}$, while the $e_{R M S}$ of the proposed LODCARC controller is around $0.08 \mu \mathrm{m}$. It also can be seen from the $e_{M}$ in Table 1 that the proposed LODCARC controller performs best.

Following this, the contouring performance of the micro-motion stage for tracking circular trajectory was analyzed. The contour tracking results during one circular motion period are depicted in Figure 5, with the corresponding contouring errors over $0.2 \mathrm{~s}$ being illustrated in Figure 6. The experimental results in terms of performance indexes are given in Table 2, where $\varepsilon_{R M S}$ is the root mean square value of the contouring error and $\varepsilon_{M}$ is the maximum value of the contouring error. 


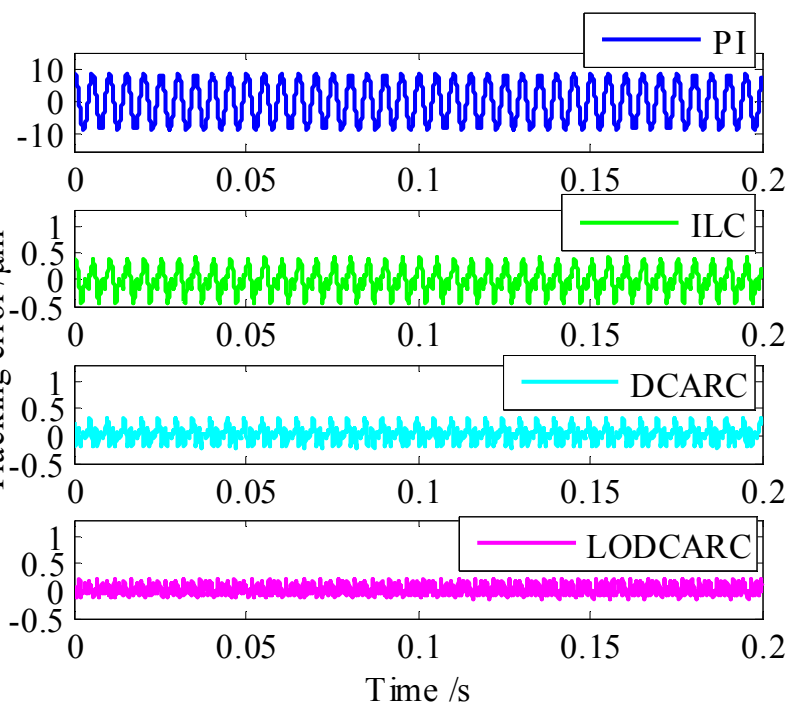

Figure 4. Axis tracking errors of circular trajectory in the $X$-axis.

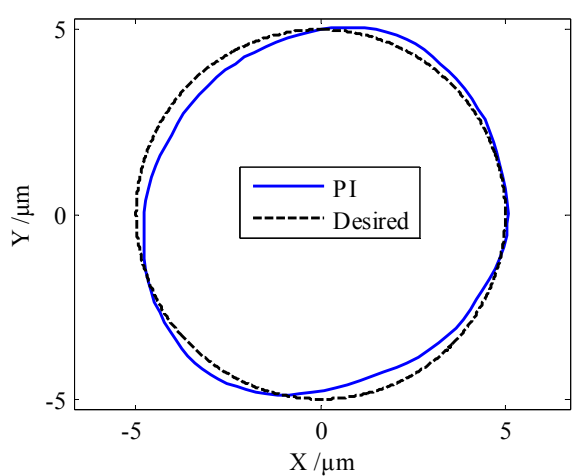

(a)

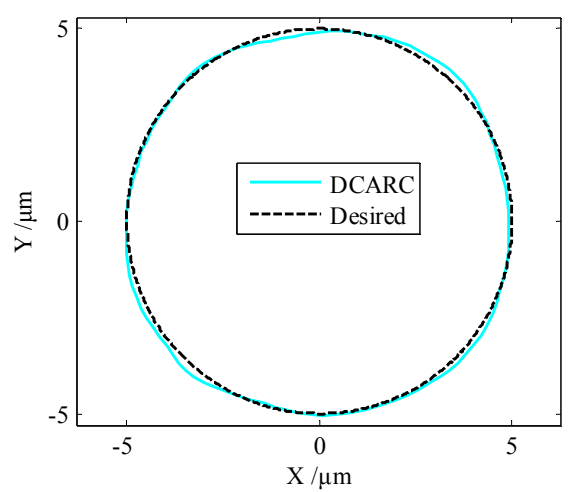

(c)

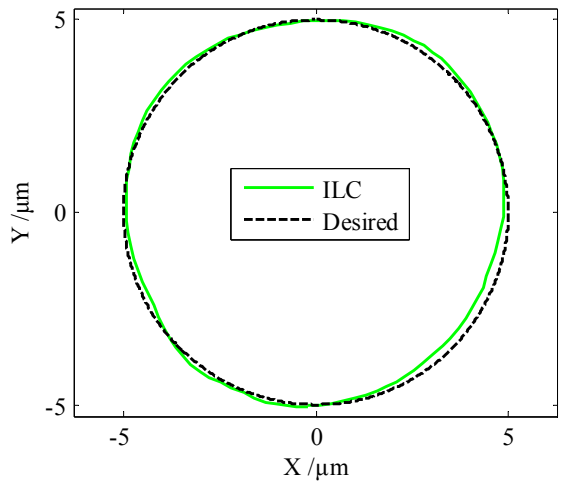

(b)

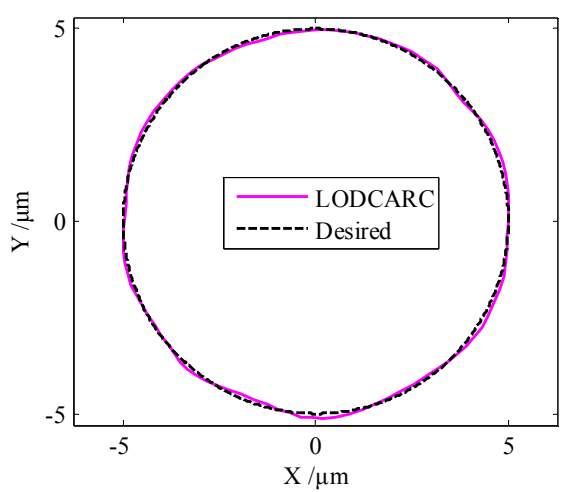

(d)

Figure 5. Contouring performances of circular trajectory using (a) PI (proportional-integral) controller; (b) ILC (iterative learning control) controller; (c) DCARC (desired compensation adaptive robust control) controller; and (d) LODCARC (learning-based optimal desired compensation adaptive robust control) controller. 


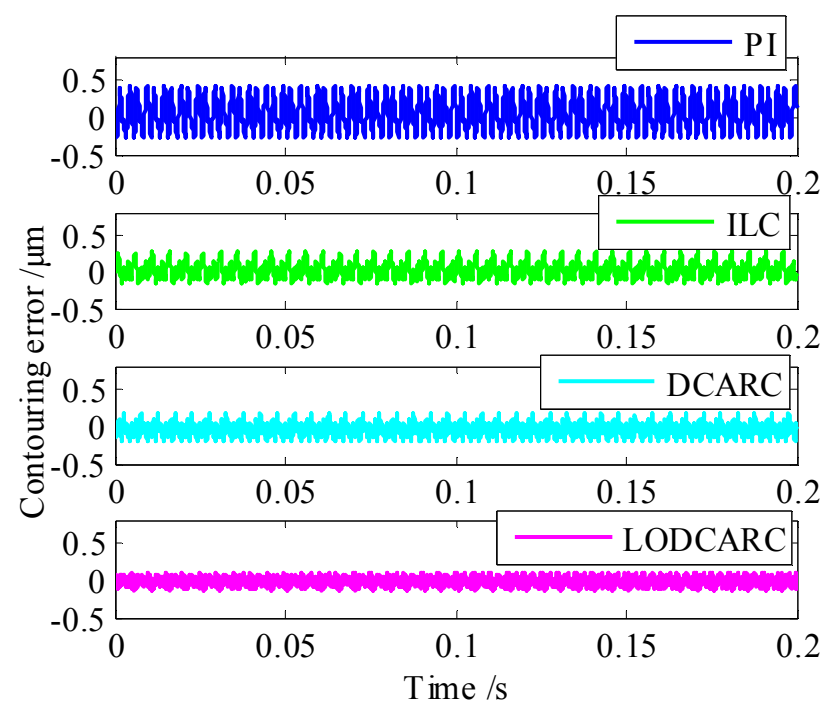

Figure 6. Contouring errors of circular trajectory.

Table 1. Tracking performance indexes of circular trajectory in the $X$-axis

\begin{tabular}{ccccc}
\hline Controller $^{\mathbf{1}}$ & PI & ILC & DCARC & LODCARC \\
\hline$e_{R M S}(\mu \mathrm{m})$ & 6.048 & 0.223 & 0.139 & 0.088 \\
$e_{M}(\mu \mathrm{m})$ & 8.499 & 0.436 & 0.341 & 0.217 \\
\hline
\end{tabular}

${ }^{1}$ PI: proportional-integral controller; ILC: iterative learning control controller; DCARC: desired compensation adaptive robust control controller; LODCARC: learning-based optimal desired compensation adaptive robust control controller.

Table 2. Contouring performance indexes of circular trajectory.

\begin{tabular}{ccccc}
\hline Controller & PI & ILC & DCARC & LODCARC \\
\hline$\varepsilon_{R M S}(\mu \mathrm{m})$ & 0.230 & 0.117 & 0.096 & 0.066 \\
$\varepsilon_{M}(\mu \mathrm{m})$ & 0.429 & 0.265 & 0.200 & 0.146 \\
\hline
\end{tabular}

It is clearly demonstrated from Figure 5 that all controllers can achieve good contouring performance. According to the contouring errors of all four controllers plotted in Figure 6 and the performance indexes given in Table 2, the contouring error of the PI controller is much larger than that of the other three controllers, with the $\varepsilon_{R M S}$ of PI controller being over $0.2 \mu \mathrm{m}$. The proposed LODCARC controller achieved an $\varepsilon_{R M S}$ of 0.066 , which is much smaller than the others. It can be subsequently obtained that the $\varepsilon_{R M S}$ of the proposed LODCARC controller is $29 \%$ of the $\varepsilon_{R M S}$ of PI controller, $56 \%$ of the $\varepsilon_{R M S}$ of ILC controller and $69 \%$ of the $\varepsilon_{R M S}$ of the DCARC controller. Furthermore, the $\varepsilon_{M}$ of all four controllers illustrated in Table 2 also shows that the proposed LODCARC controller outperforms the other controllers with respect to contouring performance.

\subsubsection{High-Speed Diamond-Shaped Contouring Trajectory}

To validate the performance of the proposed control strategy sufficiently, a diamond-shaped contouring trajectory with a side length of $5 \mu \mathrm{m}$ and a frequency of $50 \mathrm{~Hz}$ was also tested on the manipulator. For non-smooth trajectory, velocity planning is needed, and the S-shape curve [30] was adopted to plan the motion of each side of the diamond-shaped trajectory. Similarly, the contouring results during one motion period are shown in Figure 7, and the contouring errors over $0.2 \mathrm{~s}$ are given in Figure 8 while the corresponding performance indexes are compared in Table 3. 


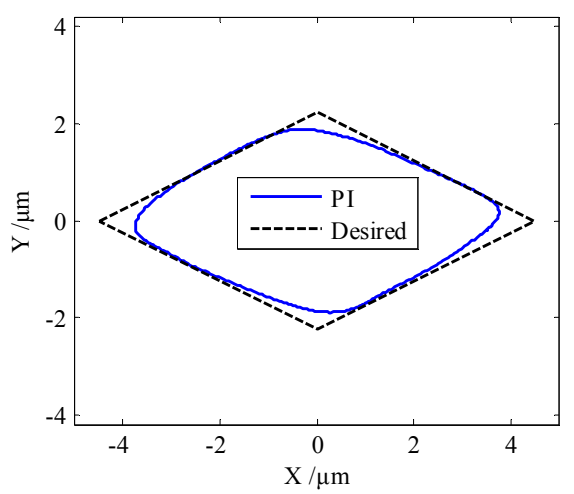

(a)

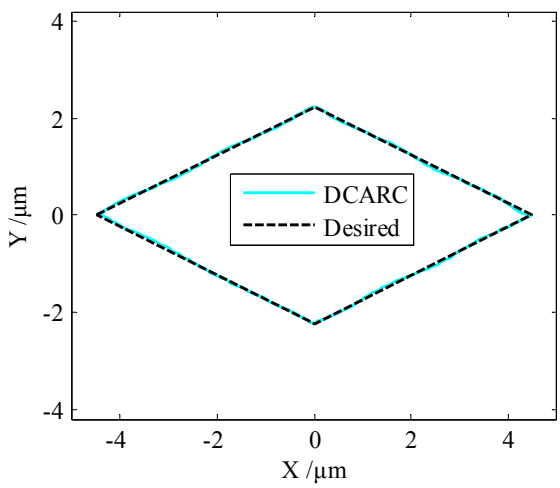

(c)

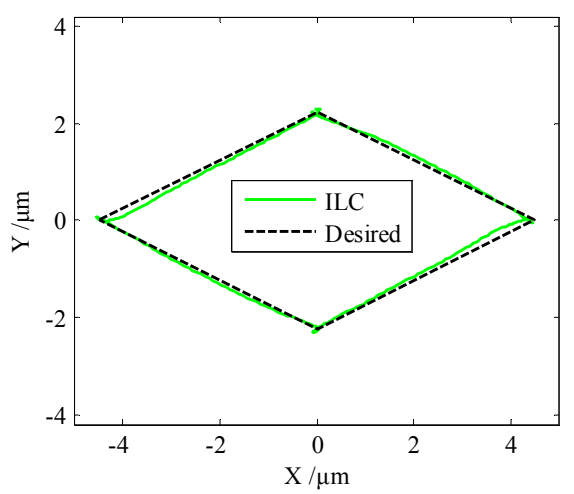

(b)

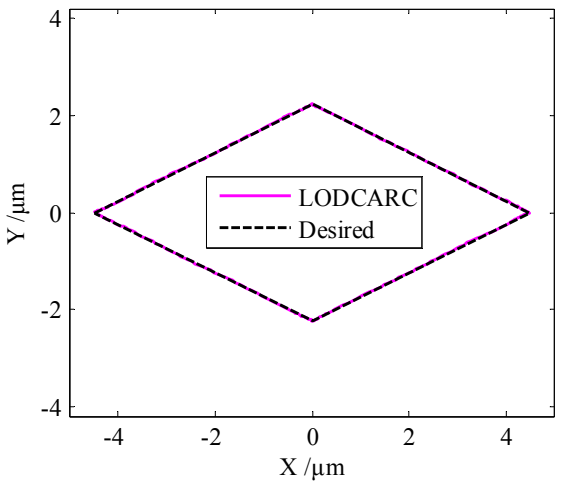

(d)

Figure 7. Contouring performances of diamond-shaped trajectory using (a) PI controller; (b) ILC controller; (c) DCARC controller; and (d) LODCARC controller.

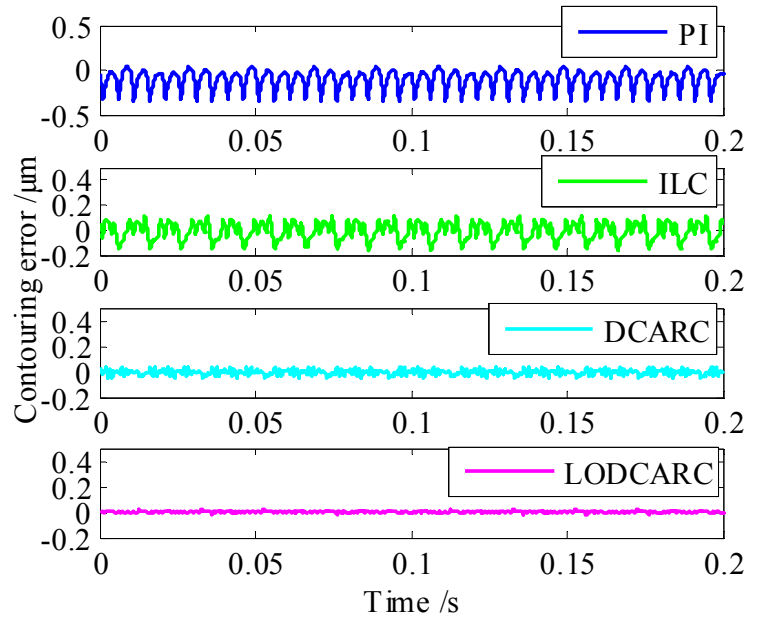

Figure 8. Contouring errors of diamond-shaped trajectory.

Table 3. Contouring performance indexes of diamond-shaped trajectory.

\begin{tabular}{ccccc}
\hline Controller & PI & ILC & DCARC & LODCARC \\
\hline$\varepsilon_{R M S}(\mu \mathrm{m})$ & 0.143 & 0.069 & 0.021 & 0.007 \\
$\varepsilon_{M}(\mu \mathrm{m})$ & 0.349 & 0.157 & 0.054 & 0.021 \\
\hline
\end{tabular}

The results in Figure 7 show that there are significant contouring errors when the PI controller and the ILC controller are adopted; the contouring performance of the PI controller is especially 
poor at the corners of the diamond-shaped trajectory. As shown in Figure 8, the contouring errors of the DCARC controller and the LODCARC controller are very small, while for LODCARC, the reference trajectory optimization compensate the effect of repetitive disturbance and uncertainty, so the contouring performance of LODCARC is greatly improved. It is clearly shown in Table 3 that both the $\varepsilon_{R M S}$ and $\varepsilon_{M}$ of the proposed LODCARC are the smallest, the $\varepsilon_{R M S}$ of the proposed LODCARC controller is $33 \%$ of the $\varepsilon_{R M S}$ of the DCARC controller, and the $\varepsilon_{M}$ of the proposed LODCARC controller is $39 \%$ of the $\varepsilon_{M}$ of DCARC controller, which also verifies the effectiveness of the proposed LODCARC control strategy.

\section{Conclusions}

In this paper, we present a learning-based optimal desired compensation adaptive robust control (LODCARC) for guaranteeing the performance of a flexure-based micro-motion manipulator. The dynamics of the flexure-based system were studied first, with proposed control strategy then being designed based on the dynamics of the system. The proposed LODCARC control algorithm possesses good parametric adaptation ability and robustness for nonlinear uncertainty, measurement noise and disturbance. This is especially the case with the help of a reference optimization method based on ILC, resulting in an effective reduction in the negative effects of repetitive unmodeled uncertainty and disturbance. Comparative experiments have been conducted on the flexure-based system, with the experimental results showing that the proposed control strategy can greatly reduce the tracking and contouring errors. Thus, our study demonstrates that the proposed LODCARC control strategy can provide a great technology for flexure-based stages to achieve excellent high-speed tracking and contouring performances.

Acknowledgments: This work was supported by the National Natural Science Foundation of China (No. 51675292).

Author Contributions: Tiemin Li, Shi Jia, and Yao Jiang proposed the method; Shi Jia, Yao Jiang, and Yunsong Du designed and performed the experiments; Tiemin Li, Shi Jia, and Yunsong Du analyzed the experimental data; Shi Jia wrote the paper.

Conflicts of Interest: The authors declare no conflicts of interest.

\section{References}

1. Kenton, B.J.; Leang, K.K. Design and control of a three-axis serial-kinematic high-bandwidth nanopositioner. IEEE ASME Trans. Mech. 2012, 17, 356-369. [CrossRef]

2. Kim, H.; Kim, J.; Ahn, D.; Gweon, D. Development of a nanoprecision 3-DOF vertical positioning system with a flexure hinge. IEEE Trans. Nanotechnol. 2013, 12, 234-245. [CrossRef]

3. Wu, J.; Wang, D.; Wang, L. A control strategy of a two degrees-of-freedom heavy duty parallel manipulator. J. Dyn. Syst. Meas. Control 2015, 137. [CrossRef]

4. Yong, Y.K.; Lu, T.; Handley, D.C. Review of circular flexure hinge design equations and derivation of empirical formulations. Precis. Eng. 2008, 32, 63-70. [CrossRef]

5. Yong, Y.K.; Moheimani, S.O.R.; Kenton, B.J.; Leang, K.K. Invited review article: High-speed flexure-guided nanopositioning: Mechanical design and control issues. Rev. Sci. Instrum. 2012, 83. [CrossRef] [PubMed]

6. Li, Y.; Xu, Q. A novel piezoactuated XY stage with parallel, decoupled, and stacked flexure structure for micro-/nanopositioning. IEEE Trans. Ind. Electron. 2011, 58, 3601-3615. [CrossRef]

7. Wu, J.; Chen, X.; Wang, L. Design and dynamics of a novel solar tracker with parallel mechanism. IEEE ASME Trans. Mech. 2016, 21, 88-97. [CrossRef]

8. Fleming, A.J.; Leang, K.K. Integrated strain and force feedback for high-performance control of piezoelectric actuators. Sens. Actuat. A Phys. 2010, 161, 256-265. [CrossRef]

9. Moheimani, S.O.R. Invited review article: Accurate and fast nanopositioning with piezoelectric tube scanners: Emerging trends and future challenges. Rev. Sci. Instrum. 2008, 79. [CrossRef] [PubMed] 
10. Croft, D.; Shed, G.; Devasia, S. Creep, hysteresis, and vibration compensation for piezoactuators: Atomic force microscopy application. In Proceedings of the American Control Conference; IEEE: New York, NY, USA, 2000; pp. 2123-2128.

11. Jiang, Y.; Li, T.; Wang, L. Stiffness modeling of compliant parallel mechanisms and applications in the performance analysis of a decoupled parallel compliant stage. Rev. Sci. Instrum. 2015, 86. [CrossRef] [PubMed]

12. Lai, L.; Gu, G.; Zhu, L. Design and control of a decoupled two degree of freedom translational parallel micro-positioning stage. Rev. Sci. Instrum. 2012, 83. [CrossRef] [PubMed]

13. Kuhnen, K. Modeling, identification and compensation of complex hysteretic nonlinearities: A modified prandti-ishlinskii approach. Eur. J. Control. 2003, 9, 407-418. [CrossRef]

14. Ge, P.; Jouaneh, M. Tracking control of a piezoceramic actuator. IEEE Trans. Control Syst. Technol. 1996, 4, 209-216.

15. Song, J.; Kiureghian, A.D. Generalized bouc-wen model for highly asymmetric hysteresis. J. Eng. Mech. 2006, 132, 610-618. [CrossRef]

16. De Lacalle, L.; Lamikiz, A.; Sanchez, J.A.; de Bustos, I.F. Simultaneous measurement of forces and machine tool position for diagnostic of machining tests. IEEE Trans. Instrum. Meas. 2005, 54, 2329-2335.

17. Uriarte, L.; Azcarate, S.; Herrero, A.; de Lacalle, L.N.L.; Lamikiz, A. Mechanistic modelling of the micro end milling operation. Proc. Inst. Mech. Eng. B J. Eng. Manuf. 2008, 222, 23-33. [CrossRef]

18. Bristow, D.A.; Tharayil, M.; Alleyne, A.G. A survey of iterative learning control. IEEE Control Syst. 2006, 26, 96-114. [CrossRef]

19. Leang, K.K.; Devasia, S. Iterative Feedforward Compensation of Hysteresis in Piezo Positioners; IEEE: New York, NY, USA, 2003; pp. 2626-2631.

20. Yao, B.; Tomizuka, M. Adaptive robust control of SISO nonlinear systems in a semi-strict feedback form. Automatica 1997, 33, 893-900. [CrossRef]

21. Hu, C.; Hu, Z.; Zhu, Y.; Wan, Z. Advanced GTCF based LARC contouring motion control on an industrial $\mathrm{X}-\mathrm{Y}$ linear motor stage with experimental investigation. IEEE Trans. Ind. Electron. 2016. [CrossRef]

22. Hu, C.; Wang, Z.; Zhu, Y.; Zhang, M.; Liu, H. Performance-oriented precision LARC Tracking motion control of a magnetically levitated planar motor with comparative experiments. IEEE Trans. Ind. Electron. 2016, 63, 5763-5773. [CrossRef]

23. Bu, F.P.; Yao, B. Desired compensation adaptive robust control of single-rod electro-hydraulic actuator. In Proceedings of the American Control Conference; IEEE: New York, NY, USA, 2001; pp. 3926-3931.

24. Yao, B.; Hu, C.; Lu, L.; Wang, Q. Adaptive robust precision motion control of a high-speed industrial gantry with cogging force compensations. IEEE Trans. Control Syst. Technol. 2011, 19, 1149-1159. [CrossRef]

25. Du, Y.; Li, T.; Jiang, Y.; Wang, H. Design and analysis of a 2-degree-of-freedom flexure-based micro-motion stage. Adv. Mech. Eng. 2016, 8. [CrossRef]

26. Jiang, Y.; Li, T.; Wang, L. Design, development and application of a compact flexure-based decoupler with high motion transmission efficiency and excellent input decoupling performance. IEEE ASME Trans. Mech. 2016. [CrossRef]

27. Qin, Y.; Shirinzadeh, B.; Tian, Y.; Zhang, D. Design issues in a decoupled XY stage: Static and dynamics modeling, hysteresis compensation, and tracking control. Sens. Actuators A Phys. 2013, 194, 95-105. [CrossRef]

28. Yao, B.; Tomizuka, M. Adaptive robust control of MIMO nonlinear systems in semi-strict feedback forms. Automatica 2001, 37, 1305-1321. [CrossRef]

29. Yu, D.; Yang, K.; Zhu, Y.; Li, X.; Cui, L. Nonlinear iterative learning control applied to an aerostatic X-Y planar motion stage. Proc. Inst. Mech. Eng. I J. Syst. Control Eng. 2012, 226, 1174-1182. [CrossRef]

30. Jiang, Y.; Li, T.; Wang, L. Dynamic modeling and redundant force optimization of a 2-DOF parallel kinematic machine with kinematic redundancy. Robot. Comput. Int. Manuf. 2015, 32, 1-10. [CrossRef]

(C) 2017 by the authors. Licensee MDPI, Basel, Switzerland. This article is an open access article distributed under the terms and conditions of the Creative Commons Attribution (CC BY) license (http:/ / creativecommons.org/licenses/by/4.0/). 